\title{
A Note on a Generalized Discounted Penalty Function in a Sparre Andersen Risk Model Perturbed by Diffusion
}

\author{
Chaolin Liu and Zhimin Zhang \\ College of Mathematics and Statistics, Chongqing University, Chongqing 401331, China \\ Correspondence should be addressed to Zhimin Zhang; cquzzm@163.com
}

Received 17 September 2013; Accepted 6 November 2013

Academic Editor: Bernhard Ruf

Copyright ( 2013 C. Liu and Z. Zhang. This is an open access article distributed under the Creative Commons Attribution License, which permits unrestricted use, distribution, and reproduction in any medium, provided the original work is properly cited.

We consider a Sparre Andersen risk model perturbed by diffusion where the interclaim times are generalized Erlang $(n)$ distribution. Generalized discounted penalty functions incorporating the maximum surplus before ruin are studied. We derive the integrodifferential equations and give the solutions for the generalized discounted penalty functions.

\section{Introduction}

Consider the following Sparre Andersen risk model perturbed by a Brownian motion:

$$
U(t)=u+c t-\sum_{i=1}^{N(t)} X_{i}+\sigma B(t)
$$

where $u \geq 0$ is the initial surplus and $c>0$ is the premium rate collected per unit time. $\left\{X_{1}, X_{2}, \ldots\right\}$, representing the individual claim amounts, is a sequence of independent and identically distributed (i.i.d.) random variables distributed like a strictly positive variable $X$ with cumulative distribution function (c.d.f.) $F(x)=\mathbb{P}(X \leq x)$, probability density function (p.d.f.) $f(x)$, and Laplace transform $\widehat{f}(s)=$ $\int_{0}^{\infty} e^{-s x} f(x) d x .\{N(t), t \geq 0\}$, a renewal process counting the number of claims up to time $t$, is defined by $N(t)=\sup \{n$ : $\left.V_{1}+\cdots+V_{n} \leq t\right\}$, where $\left\{V_{i}\right\}_{i=1}^{\infty}$ denoting the interclaim time random variables are i.i.d. like a generic variable $V$ with c.d.f. $K$, p.d.f. $k$, and Laplace transform $\widehat{k}$. Finally, $\{B(t), t \geq 0\}$ is a standard Brownian motion starting from 0 , and $\sigma>0$ is the dispersion parameter. We assume that $\left\{X_{i}\right\},\left\{V_{i}\right\}$, and $\{B(t)\}$ are mutually independent and that $c \mathbb{E} V>\mathbb{E} X$ is a net profit condition.

Let $T=\inf \{t \geq 0: U(t)<0\}$ be the ruin time with the understanding that $T=\infty$ if $U(t) \geq 0$ for all $t \geq 0$; that is, ruin does not occur. Two ruin-related quantities of interest in ruin theory are the surplus immediately before ruin $U(T-)$ and the deficit at ruin $|U(T)|$. A unified tool to study these ruin quantities is the Gerber-Shiu discounted penalty function. Recently, some researchers are interested in generalizing the Gerber-Shiu function by incorporating other quantities. One generalization is to consider the maximum surplus prior to ruin, namely, $\bar{U}(T)=\sup _{0 \leq t<T} U(t)$, and this results in the following generalized discounted penalty function:

$$
\begin{gathered}
\phi_{123}(u)=\mathbb{E}\left[e^{-\delta T} w_{123}(U(T-),|U(T)|, \bar{U}(T))\right. \\
\times I(T<\infty) \mid U(0)=u],
\end{gathered}
$$

where $\delta \geq 0$ is the interest force, $w_{123}\left(x_{1}, x_{2}, x_{3}\right): \mathbb{R}_{+}^{3} \rightarrow \mathbb{R}_{+}$ is a measurable function satisfying some integrability conditions, and $I(A)$ is the indicator function of event $A$.

In this paper, we are interested in the specific penalty function

$$
w_{123}\left(x_{1}, x_{2}, x_{3}\right)=w\left(x_{1}, x_{2}\right) I\left(x_{3}<b\right),
$$

where $b>0$ and $w\left(x_{1}, x_{2}\right): \mathbb{R}_{+}^{2} \rightarrow \mathbb{R}_{+}$is a measurable function. Thus, $\phi_{123}(u)$ reduces to the following generalized discounted penalty function for $0 \leq u<b$ :

$$
\begin{aligned}
\phi(u ; b)=\mathbb{E}\left[e^{-\delta T} w(U(T-),|U(T)|)\right. \\
\quad \times I(\bar{U}(T)<b, T<\infty) \mid U(0)=u] .
\end{aligned}
$$


Note that ruin can be caused by either a claim or oscillation of the Brownian motion. Set $w(0,0)=1$ without loss of generality. We can decompose $\phi(u ; b)$ as

$$
\phi(u ; b)=\phi_{w}(u ; b)+\phi_{d}(u ; b),
$$

where

$$
\begin{aligned}
& \phi_{w}(u ; b) \\
& =\mathbb{E}\left[e^{-\delta T} w(U(T-),|U(T)|)\right. \\
& \times I(\bar{U}(T)<b, U(T)<0, T<\infty) \mid U(0)=u], \\
& \phi_{d}(u ; b) \\
& =\mathbb{E}\left[e^{-\delta T} I(\bar{U}(T)<b, U(T)=0, T<\infty) \mid U(0)=u\right]
\end{aligned}
$$

are, respectively, the discounted penalty functions caused by a claim and oscillation of the Brownian motion.

Let $b \rightarrow \infty$; then $\phi(u ; b), \phi_{w}(u ; b)$, and $\phi_{d}(u ; b)$ reduce to the original discounted penalty functions, denoted by $\phi(u ; \infty), \phi_{w}(u ; \infty)$, and $\phi_{d}(u ; \infty)$, which have been well studied by Li and Garrido [1].

The marginal distribution of $\bar{U}(T)$ has been studied by $\mathrm{Li}$ and Dickson [2] in a Sparre Andersen risk model and Li and Lu [3] in a Markov-modulated risk model. Recently, Cheung and Landriault [4] have studied the generalized discounted penalty function $\phi_{123}(u)$ in the MAP risk model. In this paper, we focus on the evaluation of the generalized discounted penalty functions $\phi_{w}(u ; b)$ and $\phi_{d}(u ; b)$. In Section 2 , we show that $\phi_{w}(u ; b)$ and $\phi_{d}(u ; b)$ satisfy some integrodifferential equations with boundary conditions. The solutions of the integrodifferential equations will be studied in Section 3. We show that $\phi_{w}(u ; b)$ and $\phi_{d}(u ; b)$ can be expressed via $\phi_{w}(u ; \infty)$ and $\phi_{d}(u ; \infty)$ and the solutions of a homogeneous integrodifferential equation.

\section{Integrodifferential Equations}

In this section, we show that $\phi_{w}(u ; b)$ and $\phi_{d}(u ; b)$ satisfy some integrodifferential equations with boundary conditions. Before presenting our main results, we need some preliminaries.

2.1. Preliminaries. Consider a spectrally negative Lévy process $\{X(t), t \geq 0\}$ defined on $\left\{\Omega, \mathscr{F}, \mathbb{F}=\left\{\mathscr{F}_{t}\right\}_{t \geq 0}, \mathbb{P}\right\}$ which satisfies the usual conditions. Let $\mathbb{P}_{X}$ and $\mathbb{E}_{x}$ be shorthand for $\mathbb{P}(\cdot \mid X(0)=x)$ and $\mathbb{E}(\cdot \mid X(0)=x)$ with the understanding that $\mathbb{P}_{0}=\mathbb{P}$ and $\mathbb{E}_{0}=\mathbb{E}$ for the special case $x=0$.

The Laplace exponent of $X(t)$ is defined as

$$
\psi(\beta)=\frac{1}{t} \ln \mathbb{E}\left[e^{\beta X(t)}\right],
$$

which is finite at least on the positive half axis since $X(t)$ does not have positive jumps. Furthermore, $\psi(\beta)$ is convex and $\lim _{\beta \rightarrow \infty} \psi(\beta)=\infty$. Define the right inverse

$$
\Phi(q)=\sup \{\beta \geq 0: \psi(\beta)=q\},
$$

for each $q \geq 0$.
For $q \geq 0$, the scale function $W^{(q)}(x): \mathbb{R} \rightarrow[0, \infty)$ is defined as the continuous function on $[0, \infty)$ such that

$$
\int_{0}^{\infty} e^{-s x} W^{(q)}(x) d x=\frac{1}{\psi(\beta)-q}, \quad \beta>\Phi(q),
$$

and $W^{(q)}(x)=0$ for $x<0$. For $q \geq 0$, define the function $Z^{(q)}(x)$ by

$$
Z^{(q)}(x)=1+q \int_{0}^{x} W^{(q)}(y) d y
$$

with $Z^{(q)}(x)=1$ for $x \leq 0$.

For $x \in \mathbb{R}$, define

$$
\tau_{x}^{+}=\inf \{t>0: X(t)>x\}, \quad \tau_{x}^{-}=\inf \{t>0: X(t)<x\} .
$$

The scale functions play an important role in studying the one-sided and two-sided exit problems for spectrally negative Lévy process (see, e.g., Section 8 of [5]). By formula (8.9) of [5], we have for $x \leq a$ and $q \geq 0$

$$
\begin{aligned}
Q^{(q)}(x ; a) & :=\mathbb{E}_{x}\left[e^{-q \tau_{0}^{-}} I\left(\tau_{0}^{-}<\tau_{a}^{+}\right)\right] \\
& =Z^{(q)}(x)-Z^{(q)}(a) \frac{W^{(q)}(x)}{W^{(q)}(a)} .
\end{aligned}
$$

The $q$-potential measure killed on exiting $[0, a]$ is defined as

$$
R^{(q)}(x, d y ; a)=\int_{0}^{\infty} e^{-q t} \mathbb{P}_{x}\left(X(t) \in d y, t<\tau_{0}^{-} \wedge \tau_{a}^{+}\right) d t,
$$

for $q \geq 0$, where $\tau_{0}^{-} \wedge \tau_{a}^{+}=\min \left\{\tau_{0}^{-}, \tau_{a}^{+}\right\}$. By Theorem 8.7 of [5], we know that $R^{(q)}(x, d y ; a)$ has a density $r^{(q)}(x, y ; a)$ given by

$$
r^{(q)}(x, y ; a)=\frac{W^{(q)}(x) W^{(q)}(a-y)}{W^{(q)}(a)}-W^{(q)}(x-y),
$$

for $x, y \in[0, a]$

We will reproduce formulae (12) and (14) when $X(t)$ is a Brownian motion with drift; that is, $X(t)=c t+\sigma B(t)$. In this case, we have

$$
\psi(\beta)=c \beta+\frac{1}{2} \sigma^{2} \beta^{2}, \quad \Phi(q)=-\frac{c}{\sigma^{2}}+\sqrt{\frac{c^{2}}{\sigma^{4}}+\frac{2 q}{\sigma^{2}}} .
$$

Inverting the Laplace transform (9) gives

$$
W^{(q)}(x)=\frac{e^{-q_{1} x}-e^{-q_{2} x}}{\left(\sigma^{2} / 2\right)\left(q_{2}-q_{1}\right)},
$$

where $q_{1}=c / \sigma^{2}-\sqrt{c^{2} / \sigma^{4}+2 q / \sigma^{2}}, q_{2}=c / \sigma^{2}+$ $\sqrt{c^{2} / \sigma^{4}+2 q / \sigma^{2}}$. Then, by (10), we have

$$
Z^{(q)}(x)=\frac{q_{2} e^{-q_{1} x}-q_{1} e^{-q_{2} x}}{q_{2}-q_{1}} .
$$


By (16) and (17), we can reproduce (12) and (14) as follows:

$$
\begin{gathered}
Q^{(q)}(x ; a)=\frac{e^{-q_{2} x-q_{1} a}-e^{-q_{1} x-q_{2} a}}{e^{-q_{1} a}-e^{-q_{2} a}}, \quad x \leq a, \\
r^{(q)}(x, y ; a)=\frac{\left(e^{-q_{1} x-q_{2} a}-e^{-q_{2} x-q_{1} a}\right)\left(e^{q_{1} y}-e^{q_{2} y}\right)}{\left(\sigma^{2} / 2\right)\left(q_{2}-q_{1}\right)\left(e^{-q_{1} a}-e^{-q_{2} a}\right)}, \\
0 \leq y<x \leq a, \\
r^{(q)}(x, y ; a)=\frac{\left(e^{-q_{1} x}-e^{-q_{2} x}\right)\left(e^{-q_{1}(a-y)}-e^{-q_{2}(a-y)}\right)}{\left(\sigma^{2} / 2\right)\left(q_{2}-q_{1}\right)\left(e^{-q_{1} a}-e^{-q_{2} a}\right)}, \\
0 \leq x \leq y \leq a .
\end{gathered}
$$

2.2. Main Results. For $q>0$, denote by $\mathbf{e}_{q}$ an exponential r.v. with mean $1 / q$. Then, the interclaim generic variable $V$ can be expressed as $V=\mathbf{e}_{\lambda_{1}}+\cdots+\mathbf{e}_{\lambda_{n}}$, where $\mathbf{e}_{\lambda_{1}}, \ldots, \mathbf{e}_{\lambda_{n}}$ are mutually independent. Let $\mathscr{I}$ be the identity operator.

Now, we first consider the generalized discounted penalty function $\phi_{d}(u ; b)$.

Theorem 1. For $0<u<b, \phi_{d}(u ; b)$ is differentiable at least $2 n$ times and satisfies the following integrodifferential equation:

$$
\begin{gathered}
\prod_{i=1}^{n}\left(\frac{\lambda_{i}+\delta}{\lambda_{i}} \mathscr{I}-\frac{c}{\lambda_{i}} \frac{\partial}{\partial u}-\frac{\sigma^{2}}{2 \lambda_{i}} \frac{\partial^{2}}{\partial u^{2}}\right) \phi_{d}(u ; b) \\
=\int_{0}^{u} \phi_{d}(u-x ; b) f(x) d x,
\end{gathered}
$$

with boundary conditions

$$
\begin{aligned}
& \left.\prod_{i=1}^{j}\left(\frac{\lambda_{i}+\delta}{\lambda_{i}} \mathscr{I}-\frac{c}{\lambda_{i}} \frac{\partial}{\partial u}-\frac{\sigma^{2}}{2 \lambda_{i}} \frac{\partial^{2}}{\partial u^{2}}\right) \phi_{d}(u ; b)\right|_{u=0}=1, \\
& \left.\prod_{i=1}^{j}\left(\frac{\lambda_{i}+\delta}{\lambda_{i}} \mathscr{I}-\frac{c}{\lambda_{i}} \frac{\partial}{\partial u}-\frac{\sigma^{2}}{2 \lambda_{i}} \frac{\partial^{2}}{\partial u^{2}}\right) \phi_{d}(u ; b)\right|_{u=b}=0,
\end{aligned}
$$

for $j=0,1, \ldots, n-1$.

Proof. Prior to the first claim, the surplus process behaves like the Brownian motion $X(t)=u+c t+\sigma B(t)$ starting from $u$. By considering whether or not ruin occurs prior to the first claim, we have

$$
\begin{aligned}
& \phi_{d}(u ; b) \\
& =\mathbb{E}_{u}\left[e^{-\delta \tau_{0}^{-}} I\left(\tau_{0}^{-}<\tau_{b}^{+}, \tau_{0}^{-}<V\right)\right] \\
& +\int_{t \in(0, \infty)} \int_{y \in(0, b)} \int_{x \in(0, y)} e^{-\delta t} k(t) \\
& \times \mathbb{P}_{u}\left(X(t) \in d y, t<\tau_{0}^{-} \wedge \tau_{b}^{+}\right) \\
& \times \phi_{d}(y-x ; b) f(x) d x d t
\end{aligned}
$$

$$
\begin{aligned}
= & \mathbb{E}_{u}\left[e^{-\delta \tau_{0}^{-}} I\left(\tau_{0}^{-}<\tau_{b}^{+}, \tau_{0}^{-}<V\right)\right] \\
& +\int_{0}^{b} \sigma_{d}(y ; b) \mathbb{E}_{u}\left[e^{-\delta V} I\left(X(V) \in d y, V<\tau_{0}^{-} \wedge \tau_{b}^{+}\right)\right],
\end{aligned}
$$

where $\sigma_{d}(y ; b)=\int_{0}^{y} \phi_{d}(y-x ; b) f(x) d x$.

Now for $j=1, \ldots, n$, let $\mathbf{e}^{j}=\sum_{i=j}^{n} \mathbf{e}_{\lambda_{i}}$,

$$
\begin{gathered}
A_{j}(u ; b)=\mathbb{E}_{u}\left[e^{-\delta \tau_{0}^{-}} I\left(\tau_{0}^{-}<\tau_{b}^{+} \wedge \mathbf{e}^{j}\right)\right], \\
B_{j}(u, d y ; b)=\mathbb{E}_{u}\left[e^{-\delta \mathbf{e}^{j}} I\left(X\left(\mathbf{e}^{j}\right) \in d y, \mathbf{e}^{j}<\tau_{0}^{-} \wedge \tau_{b}^{+}\right)\right],
\end{gathered}
$$

and define

$$
\phi_{d, j}(u ; b)=A_{j}(u ; b)+\int_{0}^{b} \sigma_{d}(y ; b) B_{j}(u, d y ; b) .
$$

Then, we have $\phi_{d}(u ; b)=\phi_{d, 1}(u ; b)$.

For $j=1, \ldots, n-1$, we have by the Markov property,

$$
\begin{aligned}
A_{j}(u ; b)= & \mathbb{E}_{u}\left[e^{-\delta \tau_{0}^{-}} I\left(\tau_{0}^{-}<\tau_{b}^{+} \wedge \mathbf{e}_{\lambda_{j}}\right)\right] \\
& +\mathbb{E}_{u}\left[e^{-\delta \tau_{0}^{-}} I\left(\tau_{0}^{-}<\tau_{b}^{+}, \mathbf{e}_{\lambda_{j}}<\tau_{0}^{-}<\mathbf{e}^{j+1}\right)\right] \\
= & \mathbb{E}_{u}\left[e^{-\left(\lambda_{j}+\delta\right) \tau_{0}^{-}} I\left(\tau_{0}^{-}<\tau_{b}^{+}\right)\right] \\
& +\int_{0}^{\infty} \int_{0}^{b} \lambda_{j} e^{-\left(\lambda_{j}+\delta\right) t} \mathbb{P}_{u}\left(X(t) \in d x, t<\tau_{0}^{-} \wedge \tau_{b}^{+}\right) \\
& \times \mathbb{E}_{x}\left[e^{-\delta \tau_{0}^{-}} I\left(\tau_{0}^{-}<\tau_{b}^{+} \wedge \mathbf{e}^{j+1}\right)\right] d t \\
= & Q^{\left(\lambda_{j}+\delta\right)}(u ; b)+\lambda \int_{0}^{b} R^{\left(\lambda_{j}+\delta\right)}(u, d x ; b) A_{j+1}(x ; b) .
\end{aligned}
$$

Similarly, we have

$$
B_{j}(u, d y ; b)=\lambda_{j} \int_{0}^{b} R^{\left(\lambda_{j}+\delta\right)}(u, d x ; b) B_{j+1}(x, d y ; b) .
$$

Thus, (23) becomes

$$
\begin{aligned}
\phi_{d, j}(u ; b) & \\
= & Q^{\left(\lambda_{j}+\delta\right)}(u ; b) \\
& +\lambda_{j} \int_{0}^{b} R^{\left(\lambda_{j}+\delta\right)}(u, d x ; b) A_{j+1}(x ; b) \\
& +\lambda_{j} \iint_{0}^{b} \sigma_{d}(y ; b) R^{\left(\lambda_{j}+\delta\right)}(u, d x ; b) B_{j+1}(x, d y ; b) \\
= & Q^{\left(\lambda_{j}+\delta\right)}(u ; b)+\lambda_{j} \int_{0}^{b} R^{\left(\lambda_{j}+\delta\right)}(u, d x ; b) \phi_{d, j+1}(x ; b),
\end{aligned}
$$


while for $j=n$, (23) reads

$$
\begin{aligned}
\phi_{d, n}(u ; b)= & Q^{\left(\lambda_{n}+\delta\right)}(u ; b) \\
& +\lambda_{n} \int_{0}^{b} R^{\left(\lambda_{n}+\delta\right)}(u, d x ; b) \phi_{d, n+1}(x ; b),
\end{aligned}
$$

with the understanding that $\phi_{d, n+1}(x ; b):=\sigma_{d}(x ; b)$.

$$
\text { Let } q_{j, 1}=c / \sigma^{2}-\sqrt{c^{2} / \sigma^{4}+2\left(\lambda_{j}+\delta\right) / \sigma^{2}}, q_{j, 2}=c / \sigma^{2}+
$$
$\sqrt{c^{2} / \sigma^{4}+2\left(\lambda_{j}+\delta\right) / \sigma^{2}}$. Then, by formulae (18), we have for $j=1, \ldots, n$,

$$
\begin{aligned}
\phi_{d, j}(u ; b)= & \frac{e^{-q_{j, 2} u-q_{j, 1} b}-e^{-q_{j, 1} u-q_{j, 2} b}}{e^{-q_{j, 1} b}-e^{-q_{j, 2} b}} \\
& +\frac{\lambda_{j}\left(e^{-q_{j, 1} u-q_{j, 2} b}-e^{-q_{j, 2} u-q_{j, 1} b}\right)}{\left(\sigma^{2} / 2\right)\left(q_{j, 2}-q_{j, 1}\right)\left(e^{-q_{j, 1} b}-e^{-q_{j, 2} b}\right)} \\
& \times \int_{0}^{u}\left(e^{q_{j, 1} x}-e^{q_{j, 2} x}\right) \phi_{d, j+1}(x ; b) d x \\
& +\frac{\lambda_{j}\left(e^{-q_{j, 1} u}-e^{-q_{j, 2} u}\right)}{\left(\sigma^{2} / 2\right)\left(q_{j, 2}-q_{j, 1}\right)\left(e^{-q_{j, 1} b}-e^{-q_{j, 2} b}\right)} \\
& \times \int_{u}^{b}\left(e^{-q_{j, 1}(b-x)}-e^{-q_{j, 2}(b-x)}\right) \phi_{d, j+1}(x ; b) d x .
\end{aligned}
$$

From (28), we obtain the boundary conditions

$$
\phi_{d, j}(0 ; b)=1, \quad \phi_{d, j}(b ; b)=0 .
$$

Furthermore, it is readily seen from (28) that $\phi_{d, j}(u)$ is differentiable with respect to $u$ in $(0, b)$. From this fact, we can check that $\phi_{d, j}(u)$ is twice differentiable with respect to $u$ in $(0, b)$.

Note that

$$
\begin{aligned}
& \left(\frac{\partial}{\partial u}+q_{j, 1} \mathscr{I}\right)\left(\frac{\partial}{\partial u}+q_{j, 2} \mathcal{I}\right) \\
& \quad \times\left[e^{-q_{j, 1} u-q_{j, 2} b} \int_{0}^{u}\left(e^{q_{j, 1} x}-e^{q_{j, 2} x}\right) \phi_{d, j+1}(x ; b) d x\right] \\
& =\left(\frac{\partial}{\partial u}+q_{j, 2} \mathscr{I}\right)\left[\left(e^{-q_{j, 2} b}-e^{-\left(q_{j, 1}-q_{j, 2}\right) u-q_{j, 2} b}\right) \phi_{d, j+1}(u)\right], \\
& \left(\frac{\partial}{\partial u}+q_{j, 1} \mathscr{I}\right)\left(\frac{\partial}{\partial u}+q_{j, 2} \mathcal{I}\right) \\
& \quad \times\left[e^{-q_{j, 2} u-q_{j, 1} b} \int_{0}^{u}\left(e^{q_{j, 1} x}-e^{q_{j, 2} x}\right) \phi_{d, j+1}(x ; b) d x\right]
\end{aligned}
$$

$$
\begin{aligned}
&=\left(\frac{\partial}{\partial u}+q_{j, 1} \mathscr{I}\right)\left[\left(e^{-\left(q_{j, 2}-q_{j, 1}\right) u-q_{j, 1} b}-e^{-q_{j, 1} b}\right) \phi_{d, j+1}(u)\right], \\
&\left(\frac{\partial}{\partial u}+q_{j, 1} \mathscr{I}\right)\left(\frac{\partial}{\partial u}+q_{j, 2} \mathcal{I}\right) \\
& \quad \times\left[e^{-q_{j, 1} u} \int_{u}^{b}\left(e^{-q_{j, 1}(b-x)}-e^{-q_{j, 2}(b-x)}\right) \phi_{d, j+1}(x ; b) d x\right] \\
&=\left(\frac{\partial}{\partial u}+q_{j, 2} \mathscr{I}\right)\left[\left(e^{-\left(q_{j, 1}-q_{j, 2}\right) u-q_{j, 2} b}-e^{-q_{j, 1} b}\right) \phi_{d, j+1}(u)\right], \\
&\left(\frac{\partial}{\partial u}+q_{j, 1} \mathscr{I}\right)\left(\frac{\partial}{\partial u}+q_{j, 2} \mathscr{I}\right) \\
& \quad \times\left[e^{-q_{j, 2} u} \int_{u}^{b}\left(e^{-q_{j, 1}(b-x)}-e^{-q_{j, 2}(b-x)}\right) \phi_{d, j+1}(x ; b) d x\right] \\
&=\left(\frac{\partial}{\partial u}+q_{j, 1} \mathscr{I}\right)\left[\left(e^{-q_{j, 2} b}-e^{-\left(q_{j, 2}-q_{j, 1}\right) u-q_{j, 1} b}\right) \phi_{d, j+1}(u)\right] .
\end{aligned}
$$

Then, applying the operator $\left(\partial / \partial u+q_{j, 1} \mathscr{I}\right)\left(\partial / \partial u+q_{j, 2} \mathscr{I}\right)$ to both sides of (28) and using previous identities, we can obtain

$$
\phi_{d, j+1}(u ; b)=\left(\frac{\lambda_{j}+\delta}{\lambda_{j}} \mathscr{I}-\frac{c}{\lambda_{j}} \frac{\partial}{\partial u}-\frac{\sigma^{2}}{2 \lambda_{j}} \frac{\partial^{2}}{\partial u^{2}}\right) \phi_{d, j}(u ; b) .
$$

Recursively, we obtain

$$
\phi_{d, j+1}(u)=\prod_{i=1}^{j}\left(\frac{\lambda_{i}+\delta}{\lambda_{i}} \mathscr{I}-\frac{c}{\lambda_{i}} \frac{\partial}{\partial u}-\frac{\sigma^{2}}{2 \lambda_{i}} \frac{\partial^{2}}{\partial u^{2}}\right) \phi_{d}(u ; b) .
$$

In particular, by (32), the twice differentiability of $\phi_{d, n}(u ; b)$ implies that $\phi_{d}(u ; b)$ is $2 n$ times differentiable. Setting $j=n$ in (32) gives the integrodifferential equation (19). Finally, by (29) and (32), we obtain the boundary conditions (20).

Now we derive integrodifferential equation for $\phi_{w}(u)$. Similar to Theorem 1, we have the following.

Theorem 2. Let $\omega(u)=\int_{u}^{\infty} w(u, x-u) f(x) d x$. If $\omega(u)$ is differentiable, then for $0<u<b, \phi_{w}(u ; b)$ is differentiable at least $2 n$ times and satisfies the following integrodifferential equation:

$$
\begin{gathered}
\prod_{i=1}^{n}\left(\frac{\lambda_{i}+\delta}{\lambda_{i}} \mathscr{I}-\frac{c}{\lambda_{i}} \frac{\partial}{\partial u}-\frac{\sigma^{2}}{2 \lambda_{i}} \frac{\partial^{2}}{\partial u^{2}}\right) \phi_{w}(u ; b) \\
=\int_{0}^{u} \phi_{w}(u-x ; b) f(x) d x+\omega(u),
\end{gathered}
$$

with boundary conditions

$$
\begin{aligned}
& \left.\prod_{i=1}^{j}\left(\frac{\lambda_{i}+\delta}{\lambda_{i}} \mathscr{I}-\frac{c}{\lambda_{i}} \frac{\partial}{\partial u}-\frac{\sigma^{2}}{2 \lambda_{i}} \frac{\partial^{2}}{\partial u^{2}}\right) \phi_{d}(u ; b)\right|_{u=0}=0 \\
& \left.\prod_{i=1}^{j}\left(\frac{\lambda_{i}+\delta}{\lambda_{i}} \mathscr{I}-\frac{c}{\lambda_{i}} \frac{\partial}{\partial u}-\frac{\sigma^{2}}{2 \lambda_{i}} \frac{\partial^{2}}{\partial u^{2}}\right) \phi_{d}(u ; b)\right|_{u=b}=0
\end{aligned}
$$

for $j=0,1, \ldots, n-1$. 
Proof. Similar to (21), we have

$\phi_{w}(u)=\int_{0}^{b} \sigma_{w}(y ; b) \mathbb{E}_{u}\left[e^{-\delta V} I\left(X(V) \in d y, V<\tau_{0}^{-} \wedge \tau_{b}^{+}\right)\right]$,

where $\sigma_{w}(y)=\int_{0}^{y} \phi_{w}(y-x ; b) f(x) d x+\omega(y)$. The rest of the proof is exactly the same as Theorem 1 .

Remark 3. Different from Li and Garrido [1], we analyze the differentiability and derive the integrodifferential equation for the generalized discounted penalty function at the same time. Instead of using Taylor's expansion, the techniques used in the proof of Theorems 1 and 2 are based on the one-sided and two-sided exit results in Lévy process. We remark that such techniques have also been successfully used in analyzing the dependent risk model perturbed by diffusion (see, e.g., Zhang and Yang [6]).

Remark 4. We have significantly relaxed the condition on the $2 n$ times differentiability of the Gerber-Shiu functions presented in Propositions 2 and 4 of Li and Garrido [1], where the twice differentiability of $\omega(u)$ and $f(x)$ has been assumed.

\section{The Solutions}

In this section, we derive the solutions of the integrodifferential equations (19) and (33).

We relax the restriction $0<u<b$ to $u>0$ in equations (19) and (33) and note by Theorem 1 of Li and Garrido [1] that

$$
\begin{gathered}
\prod_{i=1}^{n}\left(\frac{\lambda_{i}+\delta}{\lambda_{i}} \mathscr{I}-\frac{c}{\lambda_{i}} \frac{\partial}{\partial u}-\frac{\sigma^{2}}{2 \lambda_{i}} \frac{\partial^{2}}{\partial u^{2}}\right) \phi_{d}(u ; \infty) \\
=\int_{0}^{u} \phi_{d}(u-x ; \infty) f(x) d x, \\
\prod_{i=1}^{n}\left(\frac{\lambda_{i}+\delta}{\lambda_{i}} \mathscr{I}-\frac{c}{\lambda_{i}} \frac{\partial}{\partial u}-\frac{\sigma^{2}}{2 \lambda_{i}} \frac{\partial^{2}}{\partial u^{2}}\right) \phi_{w}(u ; \infty) \\
=\int_{0}^{u} \phi_{w}(u-x ; \infty) f(x) d x+\omega(u) .
\end{gathered}
$$

Thus, by the general theory of differential equations, we have

$$
\begin{aligned}
& \phi_{d}(u ; b)=\phi_{d}(u ; \infty)+\sum_{i=1}^{2 n} k_{d, i} v_{i}(u), \\
& \phi_{w}(u ; b)=\phi_{w}(u ; \infty)+\sum_{i=1}^{2 n} k_{w, i} v_{i}(u),
\end{aligned}
$$

where $k_{d, i}$ 's and $k_{w, i}$ 's are constants determined by the boundary conditions $(20),(34)$, and $v_{1}(u), \ldots, v_{2 n}(u)$ are linearly independent solutions of the following homogeneous integrodifferential equation:

$$
\begin{gathered}
\prod_{i=1}^{n}\left(\frac{\lambda_{i}+\delta}{\lambda_{i}} \mathscr{J}-\frac{c}{\lambda_{i}} \frac{\partial}{\partial u}-\frac{\sigma^{2}}{2 \lambda_{i}} \frac{\partial^{2}}{\partial u^{2}}\right) v(u) \\
=\int_{0}^{u} v(u-x) f(x) d x, \quad u \geq 0
\end{gathered}
$$

We remark that $\phi_{d}(u ; \infty)$ and $\phi_{w}(u ; \infty)$ have been well investigated by Li and Garrido [1]. If the p.d.f. $f$ has a rational Laplace transform (a ratio of two polynomials), the solutions $v_{i}(u)$ 's to the homogeneous integrodifferential equation (38) can be obtained by Laplace transforms as follows.

Assume that the claim size $X$ is rationally distributed with

$$
\widehat{f}(s)=\frac{q_{m-1}(s)}{q_{m}(s)}
$$

where $q_{m}(s)$ is a polynomial of degree $m$ without zeros in the right half complex plane and $q_{m-1}(s)$ is a polynomial of degree $m-1$ satisfying $q_{m-1}(0)=q_{m}(0)$. Assume without loss of generality that the leading coefficient of $q_{m}(s)$ is 1 .

Let

$$
B(s)=\prod_{i=1}^{n}\left(\frac{\lambda_{i}+\delta}{\lambda_{i}}-\frac{c}{\lambda_{i}} s-\frac{\sigma^{2}}{2 \lambda_{i}} s^{2}\right)=\sum_{i=0}^{2 n} B_{i} s^{i} .
$$

Taking Laplace transforms on both sides of (38) gives

$$
B(s) \widehat{v}(s)-C(s)=\widehat{v}(s) \widehat{f}(s),
$$

where $C(s)=\sum_{i=1}^{2 n} \sum_{j=0}^{i-1} B_{i} s^{j} v^{(i-1-j)}(0)$ is a polynomial of degree $2 n-1$ or less. Then (41) gives

$$
\widehat{v}(s)=\frac{C(s) q_{m}(s)}{B(s) q_{m}(s)-q_{m-1}(s)} .
$$

By Theorem 2 of [1], the denominator of (42) can be factorized as follows:

$$
B(s) q_{m}(s)-q_{m-1}(s)=D \prod_{i=1}^{n}\left(s-\rho_{i}\right) \prod_{j=1}^{n+m}\left(s+R_{j}\right)
$$

where $D=\prod_{i=1}^{n}\left(-\sigma^{2} / 2 \lambda_{i}\right)^{n}, \rho_{i}$ 's, and $-R_{j}$ 's are zeros of the denominator of (42) lying in the right and left half complex plane, respectively. If $\rho_{i}$ 's and $R_{j}$ 's are distinct, we have by partial fraction

$$
\widehat{v}(s)=\sum_{i=1}^{n} \frac{a_{i}}{s-\rho_{i}}+\sum_{j=1}^{n+m} \frac{b_{j}}{s+R_{j}}
$$

where

$$
\begin{gathered}
a_{i}=\frac{C\left(\rho_{i}\right) q_{m}\left(\rho_{i}\right)}{D \prod_{r=1, r \neq i}^{n}\left(\rho_{i}-\rho_{r}\right) \prod_{j=1}^{n+m}\left(\rho_{i}+R_{j}\right)}, \\
b_{j}=\frac{C\left(-R_{j}\right) q_{m}\left(-R_{j}\right)}{D \prod_{i=1}^{n}\left(-R_{j}-\rho_{i}\right) \prod_{r=1, r \neq j}^{n+m}\left(R_{r}-R_{j}\right)} .
\end{gathered}
$$

Upon inversion, (44) gives

$$
v(u)=\sum_{i=1}^{n} a_{i} e^{\rho_{i} u}+\sum_{j=1}^{n+m} b_{j} e^{-R j u}, \quad u \geq 0
$$


Finally, the $2 n$ linearly independent solutions $v_{1}(u), \ldots$, $v_{2 n}(u)$ can be obtained by specifying the initial conditions

$$
v_{i}^{(j)}(0)=I(j=i-1), \quad i=1, \ldots, 2 n, j=0,1, \ldots, 2 n-1,
$$

in $C(s)$.

In the rest, we pay attention to the classical compound Poisson risk model perturbed by diffusion; that is, $n=1$. From Li [7], we know that the two linearly independent solutions $v_{1}(u)$ and $v_{2}(u)$ to $(38)(n=1)$ can be chosen to be

$$
v_{1}(u)=\phi_{d}(u ; \infty), \quad v_{2}(u)=\int_{0}^{u} \phi_{d}(u-x ; \infty) e^{\rho_{1} x} d x,
$$

where $\rho_{1}$ is the solution of equation $\lambda_{1}+\delta-c s-\left(\sigma^{2} / 2\right) s^{2}-$ $\lambda_{1} \widehat{f}(s)=0$ in the right half complex plane.

By the boundary conditions

$$
\begin{array}{ll}
\phi_{d}(0 ; b)=1, & \phi_{d}(b ; b)=0, \\
\phi_{w}(0 ; b)=0, & \phi_{w}(b ; 0)=0,
\end{array}
$$

we obtain

$$
\begin{aligned}
& k_{d, 1}=0, \quad k_{d, 2}=-\frac{\phi_{d}(b ; \infty)}{\int_{0}^{b} \phi_{d}(b-x ; \infty) e^{\rho_{1} x} d x}, \\
& k_{w, 1}=0, \quad k_{w, 2}=-\frac{\phi_{w}(b ; \infty)}{\int_{0}^{b} \phi_{d}(b-x ; \infty) e^{\rho_{1} x} d x} .
\end{aligned}
$$

Then, the generalized discounted penalty functions are given by

$$
\begin{aligned}
& \phi_{d}(u ; b)=\phi_{d}(u ; \infty)-\frac{\int_{0}^{u} \phi_{d}(u-x ; \infty) e^{\rho_{1} x} d x}{\int_{0}^{b} \phi_{d}(b-x ; \infty) e^{\rho_{1} x} d x} \phi_{d}(u ; \infty), \\
& \phi_{w}(u ; b)=\phi_{w}(u ; \infty)-\frac{\int_{0}^{u} \phi_{d}(u-x ; \infty) e^{\rho_{1} x} d x}{\int_{0}^{b} \phi_{d}(b-x ; \infty) e^{\rho_{1} x} d x} \phi_{w}(u ; \infty),
\end{aligned}
$$

which implies that the generalized discounted penalty functions are proportional to the original discounted penalty functions.

\section{Acknowledgments}

The authors would like to thank the editor and the anonymous referee for their very helpful suggestions and comments. This research is supported by the Fundamental Research Funds for the Central Universities (Project no. CQDXWL-2012-001).

\section{References}

[1] S. Li and J. Garrido, "The Gerber-Shiu function in a Sparre Andersen risk process perturbed by diffusion," Scandinavian Actuarial Journal, no. 3, pp. 161-186, 2005.
[2] S. Li and D. C. M. Dickson, "The maximum surplus before ruin in an $\operatorname{Erlang}(n)$ risk process and related problems," Insurance: Mathematics \& Economics, vol. 38, no. 3, pp. 529-539, 2006.

[3] S. Li and Y. Lu, "The decompositions of the discounted penalty functions and dividends-penalty identity in a Markov-modulated risk model," Astin Bulletin, vol. 38, no. 1, pp. 53-71, 2008.

[4] E. C. K. Cheung and D. Landriault, "A generalized penalty function with the maximum surplus prior to ruin in a MAP risk model," Insurance: Mathematics \& Economics, vol. 46, no. 1, pp. 127-134, 2010.

[5] A. E. Kyprianou, Introductory Lectures on Fluctuations of Lévy Processes with Applications, Springer, Berlin, Germany, 2006.

[6] Z. Zhang and H. Yang, "Gerber-Shiu analysis in a perturbed risk model with dependence between claim sizes and interclaim times," Journal of Computational and Applied Mathematics, vol. 235, no. 5, pp. 1189-1204, 2011.

[7] S. Li, "The distribution of the dividend payments in the compound Poisson risk model perturbed by diffusion," Scandinavian Actuarial Journal, no. 2, pp. 73-85, 2006. 


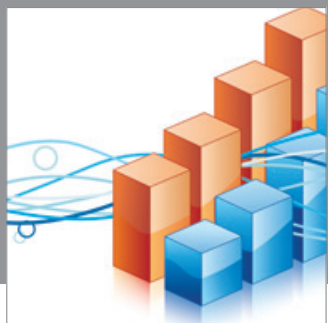

Advances in

Operations Research

mansans

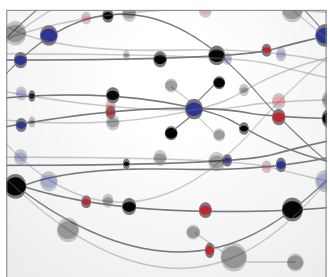

The Scientific World Journal
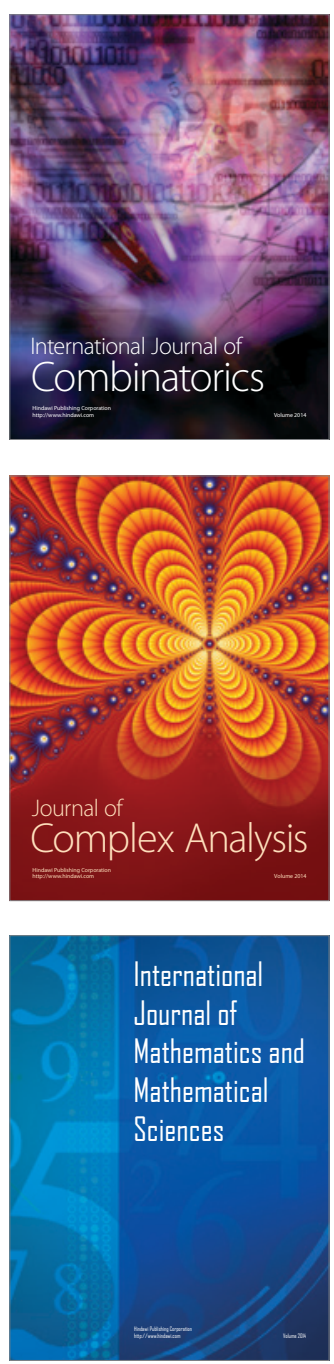
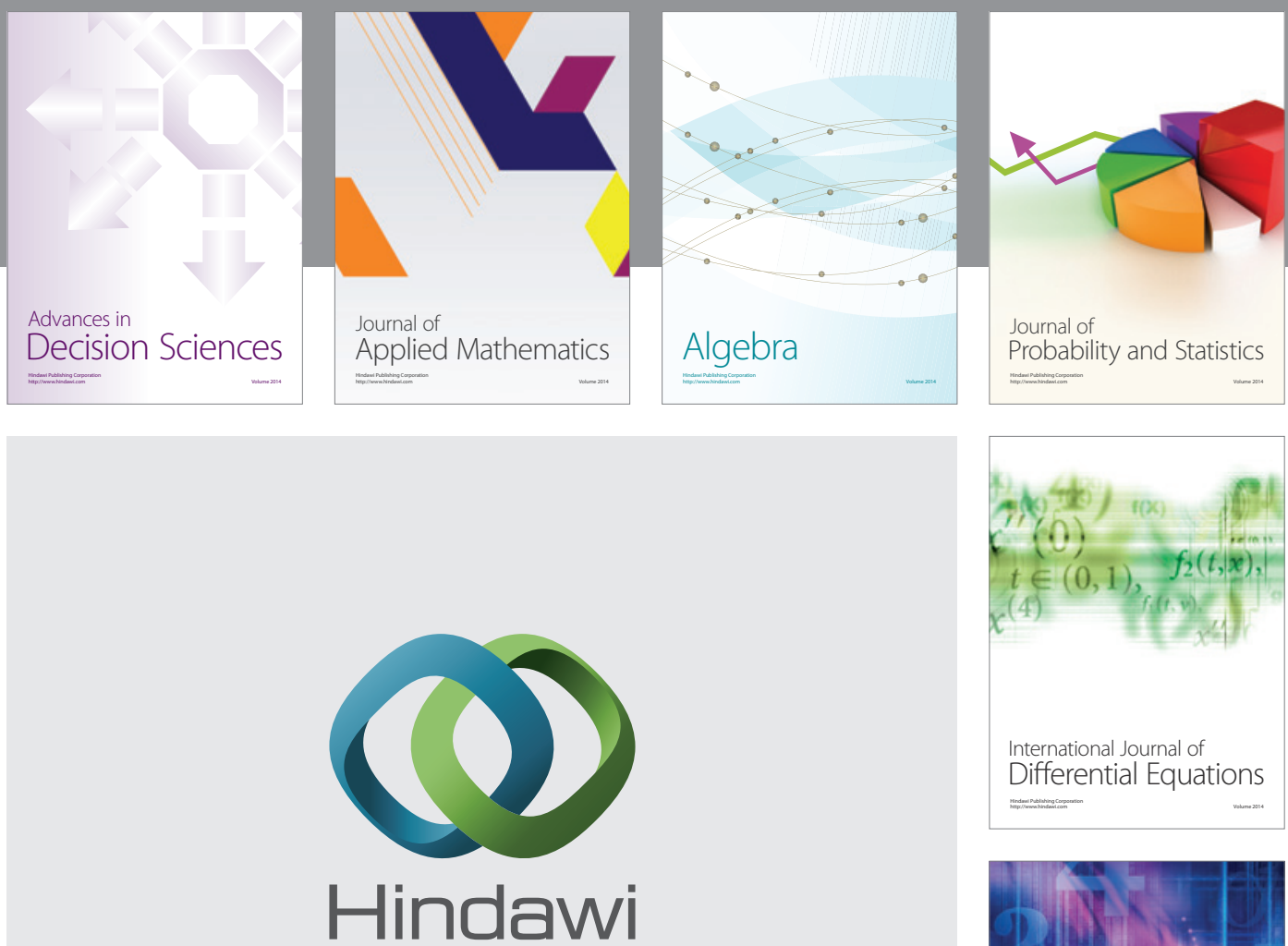

Submit your manuscripts at http://www.hindawi.com
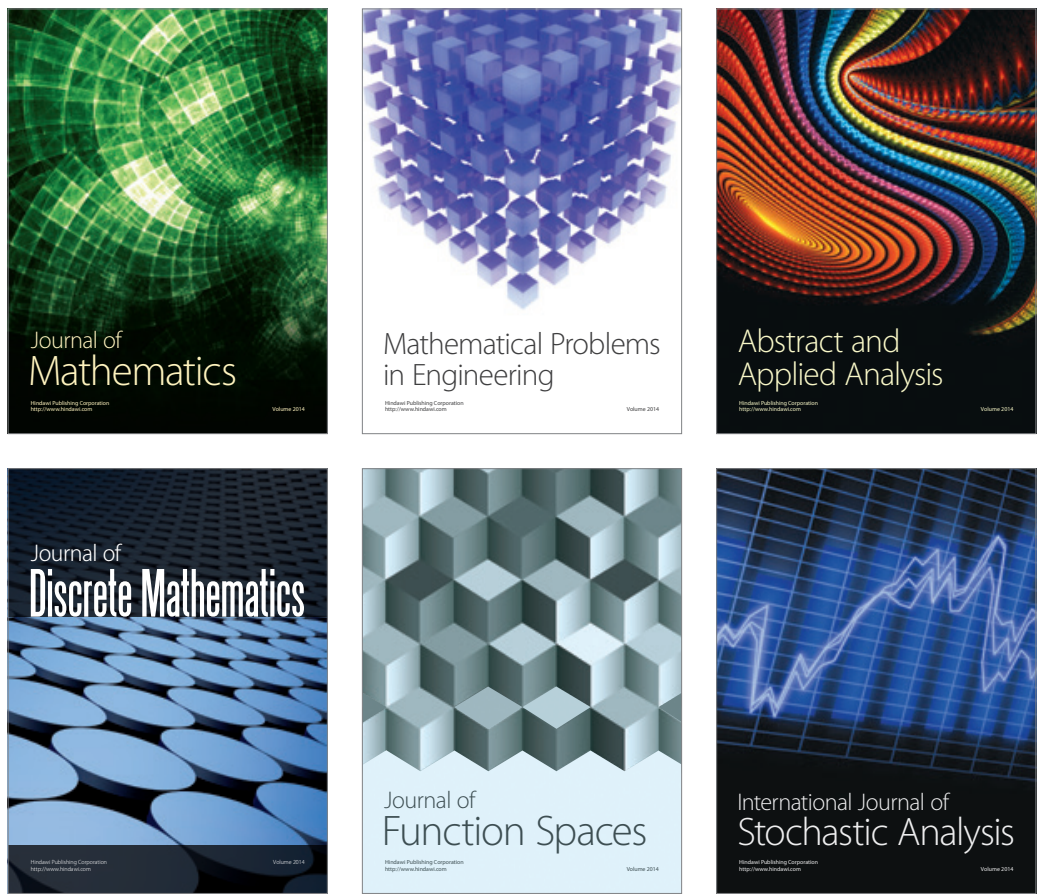

Journal of

Function Spaces

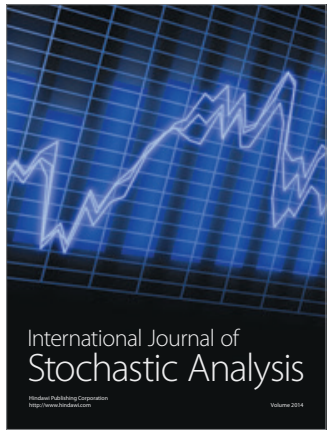

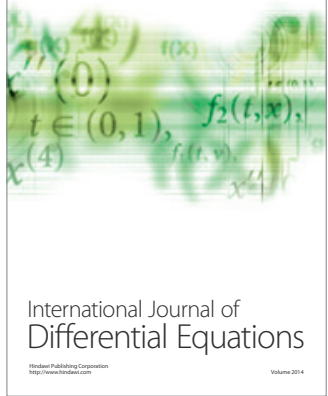
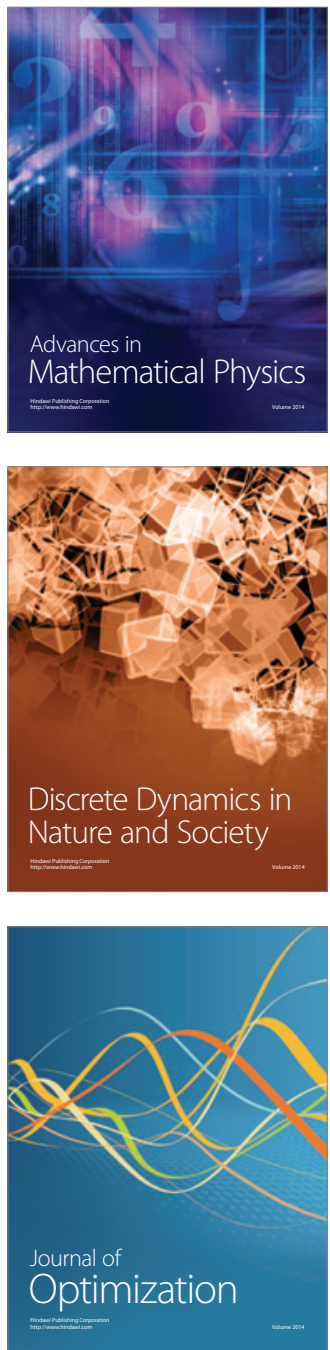